\title{
Interventions for Fetal Alcohol Spectrum Disorder: Meeting Needs Across the Lifespan
}

\author{
Jacqueline Pei ${ }^{1 *}$, Katherine Flannigan ${ }^{2}$, Lori Walls ${ }^{3}$ and Carmen Rasmussen ${ }^{4}$ \\ ${ }^{1}$ University of Alberta, Department of Educational Psychology, Edmonton, AB, Canada \\ ${ }^{2}$ University of British Columbia, Department of Pediatrics, Vancouver, BC, Canada \\ ${ }^{3}$ Sanguine Psychological Services, Toronto, ON, Canada \\ ${ }^{4}$ University of Alberta, Department of Pediatrics, Edmonton, AB, Canada
}

*Corresponding author: Jacqueline Pei, University of Alberta, Department of Educational Psychology, Edmonton, AB, Canada, Tel: 780-248-1167; Fax: 780-492-1318; E-mail: jpei@ualberta.ca

Rec date: Nov 30, 2015; Acc date: Jan 27 2016; Pub date: Feb 04, 2016

Copyright: (c) 2016 Pei J, et al. This is an open-access article distributed under the terms of the Creative Commons Attribution License, which permits unrestricted use, distribution, and reproduction in any medium, provided the original author and source are credited.

\begin{abstract}
Background: Fetal Alcohol Spectrum Disorder (FASD) is a complicated disability resulting in a diverse, highneeds population for whom intervention is critical to optimize functional competence and reduce the emergence of adverse outcomes. Researchers have been evaluating intervention efforts for FASD to inform practice and policy decision-making.

Objective: The current review provides a synopsis of the current state of evidence for intervention research in FASD, with consideration of how our growing understanding of the unique needs of individuals with FASD might inform future intervention initiatives.

Method: A comprehensive literature review was conducted across a number of databases using multiple search terms linking FASD and intervention.

Results: Existing evidence-based interventions are limited and focus predominantly on the school-aged population, thereby neglecting adolescents and adults. Future research efforts are needed to support individuals with FASD across the entire lifespan, particularly during transitions and for individuals involved in the legal system.

Conclusion: As the field of FASD continues to grow, so must the quality and quantity of intervention research. It is through cross-discipline intervention research efforts that the evidence supporting best practices will be established, and policies can be implemented to reflect best uses of available funds to support this population.
\end{abstract}

Keywords: Fetal alcohol spectrum disorder; Fetal alcohol syndrome; Fasd; Prenatal alcohol exposure; Alcohol induced disorders; Intervention

\section{Background}

Prenatal Alcohol Exposure (PAE) can lead to a variety of cognitive, behavioural and neurological deficits, including permanent structural and functional damage to the brain, which fall under the umbrella term of Fetal Alcohol Spectrum Disorder (FASD; [1]). Researchers have estimated the prevalence of FASD in North America and other industrialized countries to be between 1.4 and $4.8 \%$ in the general population [2-4]. Importantly, the rates of FASD may be higher in some developing counties and specific communities, underscoring the global impact of the disability [2]. The overall cost for Canadian individuals with FASD aged 0 to 53 years is a reported $\$ 5.3$ billion annually, with an average annual cost of $\$ 21,642$ per individual [3]. These expenses include the cost of service delivery to address needs resulting from primary brain injury, as well as costs associated with adverse outcomes such as school expulsion, trouble with the law, incarceration, and addictions [5].

An early diagnosis of FASD leading to interventions in the preschool years has been described as a protective factor and is associated with more positive outcomes in adolescence and adulthood compared to receiving a diagnosis later in life [6]. In turn, early intervention is crucial for fostering positive outcomes for individuals with FASD, such as optimizing access to supports which may protect against adverse outcomes and developing secondary disabilities [7]. However, despite the plethora of research on the challenges of FASD and the importance of early intervention, relatively few evidence-based interventions exist for this population [8-10]. Furthermore, the challenges associated with FASD have a significant influence on family dynamics and caregiver experience, which in turn has an important impact on long-term outcomes [11]. Designing appropriate interventions for individuals with FASD is extremely complex, due to the multitude of pre- and post-natal risk factors associated with the disorder [12]. Therefore, a comprehensive understanding of needs is important for informing interventions [13] because the severity, range, and areas of impairment for FASD-affected individuals are vast and highly variable [14]. That said, it may not be feasible to tailor interventions to each individual in all situations. Thus, identification of core needs of any individual with FASD and their caregivers should be considered in intervention planning. As well, understanding the ways in which those needs map to existing evidence-based interventions is an important next step: generating a more feasible approach to 'generalized individualization'. To this end, in this paper we first describe the needs of individuals with FASD and their caregivers, then pair this information with evidence-based intervention practices, and identify gaps in this knowledge. Challenges related to intervention research - as well as implementation of interventions in the community - are also identified, and a process for informed progression of practice is explored to support continued advancement of the field. 


\section{Method}

The current review was conducted by searching the following academic databases: Web of Science, PsycInfo, PubMed, ERIC, and Google Scholar. Search terms included fetal alcohol spectrum disorder/ FASD/fetal alcohol syndrome/FAS/prenatal alcohol exposure/PAE, and intervention/treatment/therapy. Reference lists from other intervention literature reviews were also searched for relevant studies.

\section{Common Needs and Related Interventions}

\section{Neurocognitive function}

\section{Needs}

Deficits in intellectual functioning, information processing, attention, executive functioning, visual perception and construction, social cognition, and learning and memory have widely been reported across the lifespan among individual with PAE and FASD [15-17].

\section{Neurocognitive interventions}

Several interventions have been directed towards improving neurocognitive function in FASD, and there is a growing evidence base for cognitive interventions targeting self-regulation, memory, and attention.

\section{Self-regulation}

Cognitive Control Therapy (CCT) was developed several decades ago and is designed to reorganize dysfunctional cognitive controls and strategies by teaching children how to think differently, and enhance self-observation and regulation [18-20]. Adnams et al. [19] piloted CCT techniques in 10 children (mean age of 8 years) with Fetal Alcohol Syndrome in classrooms in South Africa. Children in the CCT group improved significantly in terms of teacher-rated behaviours and showed a non-significant improvement in cognitive control, but no change was noted in neuropsychological test scores.

The Alert Program ${ }^{\oplus}$, developed by Williams and Shellenberger [21], is a promising self-regulation intervention that has been studied among children with FASD. Wells et al. [22] studied the efficacy of the neurocognitive habilitation intervention (based on the Alert Program ${ }^{\circledR}$ ) focusing on self-regulation among 40 children (aged 6 to 11 years) with FASD. These researchers found that the intervention led to significant improvements in emotional functioning and parent-rated executive functional behaviors in a treatment group compared to a nointervention control group with prenatal alcohol exposure $(n=38)$.

Nash et al. [23] implemented the Alert Program ${ }^{\bullet}$ with 25 children with FASD (aged 8 to 12 years), who were assigned to either an immediate treatment group or a delayed treatment control group. The authors reported that the intervention led to significant improvements in inhibitory control and social cognition, as well as higher parent ratings of behavioural and emotional regulation, and reduced externalizing behaviours, some of which were maintained at 6-month follow-up. Soh et al. [24] further examined the impact of the Alert Program $^{\circ}$ with 29 children with FASD (aged 8 to 12 years) and reported that the children showed increases in frontal grey matter in brain areas critical for self-regulation.

\section{Memory}

Loomes, Rasmussen, Pei, Manji, and Andrew [25] examined the efficacy of a brief intervention designed to teach young children how to rehearse verbal information. Young children with FASD $(n=33$, aged 4 to 11 years) were assigned to an intervention or control group, and the authors found that the intervention group improved more than the control group on a digit span working memory test after the intervention.

\section{Attention}

Kerns et al. [26] evaluated attention using a computerized training program - the Computerized Progressive Attention Training (CPAT) with 10 students (aged 8 to 15 years) with FASD, which was conducted over approximately 9 weeks. Significant improvements were reported between pre- and post-intervention assessment scores for distractibility, sustained and divided attention, working memory, and math and reading. Both parent and teacher reviews of the intervention were also positive.

\section{Gaps}

This emerging data on neurocognitive intervention reveals potentially promising approaches for supporting self-regulation, working memory, and attention among children with FASD. However, interventions targeting deficits in visual perception and construction, broader executive functions, and social cognition have yet to be examined in the literature. Additionally, the neurocognitive interventions that have been studied in FASD have primarily targeted younger children, with adolescent and adult groups absent in most studies. Also lacking in the literature is longitudinal follow-up research and replication studies to support efficacy in community-based implementation.

\section{Academics}

\section{Needs}

Students with FASD experience various academic difficulties including deficits in reading, spelling, and math [17,27-29], which can have a significant impact on their school experience and social functioning [30]. Consequently, many individuals with FASD require special education services. The academic challenges experienced by individuals with FASD may extend into adulthood, leading to high rates of unemployment and vocational disability, especially when unsupported [31].

\section{Academic interventions}

In general, researchers examining academic interventions for FASD suggest that approaches should be collaborative and incorporate multiple strategies for teaching new skills, self-monitoring and regulation, modifying the environment, and basic behavioural principles (e.g., positive reinforcement, natural consequences) [32].

\section{Mathematics}

Kable et al. [33] developed and tested a math intervention program called the Math Interactive Learning Experience (MILE) for 61 young children with FASD (aged 3 to 10 years). The MILE intervention consisted of individualized math tutoring with a focus on cognitive functions, such as working memory and visual-spatial skills, which are 
involved in mathematics. The MILE group showed more significant gains in math performance after the 6-week intervention compared to an FASD contrast group who received standard psycho-educational care, and these gains were maintained at the 6-month follow-up [34] Recent work with the MILE program has also shown that the intervention may be effectively translated into the community for administration by trained instructors [35].

\section{Language and literacy}

Researchers have also studied the effect of a language and literacy training (LLT) program for children (aged 9 years) with FASD in South Africa [19]. The authors recruited 65 children, 40 of whom were diagnosed with FASD and 25 of whom were not alcohol exposed. Children with FASD were randomly assigned to either the LLT intervention group, who participated in weekly sessions with a speech and language therapist (over 9 months), or the FASD control group. Compared with the FASD control group, the LLT group showed significantly more improvements on measures of language and literacy. However, the LLT group, FASD control group, and the non-alcohol exposed control groups showed similar changes on general scholastic achievement.

\section{Gaps}

Similar to the neurocognitive interventions reviewed above, academic interventions for individuals with FASD are showing some promising results. However, despite these positive steps, there remain important gaps in terms of examination of writing and spelling skills, and functional literacy. Moreover, all of the studies related to academic interventions have focused on younger children, and work with adolescents and adult learners is again lacking.

\section{Behaviour}

\section{Needs}

Behavioural difficulties are common in FASD and are experienced across a wide variety of settings, verified by reports from caregivers, teachers, and clinicians, as well as self-reports from affected individuals [36]. Some of the most commonly reported problems include adaptive behaviour challenges, aggression, delinquency, conduct problems, and other high risk behaviours [5], which can extend into adulthood [31]. Criminal behaviour is also a concern for this group [5], with a disproportionately high percentage of youth and adults with a FASD in correctional facilities $[37,38]$. Importantly, a major protective factor against long-term behaviour problems in FASD is access to therapeutic help and assistance [31]. To date, the interventions that specifically target behaviour problems are limited.

\section{Behavioural interventions}

Behavioural interventions for individuals with FASD often reflect a reframing of challenging behaviour by shifting our interpretation of the observed behaviours [12]. They also involve approaches to increase the capacity of caregiver or support systems to create environments that both reduce maladaptive coping on the part of the children, and build strengths [12].

\section{Families moving forward}

One strategy for addressing problematic behaviours associated with FASD is appreciating that these challenges are reflective of neurological brain damage (caused by PAE) rather than willful disobedience or noncompliance [12]. Carmichael-Olson and colleagues' Families Moving Forward (FMF) program [39] provides behavioural consultation for families and school personnel that encourages shifts in cognitions and attitudes, fosters connections to community resources, and teaches families and caregivers strategies for modifying the environment to reduce triggers for FASD-related behavioural problems [12]. Early examination of this approach among children with FASD (aged 5 to 11 years) and their caregivers $(n=52)$ revealed that, after 16 bi-weekly sessions of 90 minutes, caregivers in the FMF intervention reported significant improvement in self-efficacy and engagement in self-care practices, and reduced problem behaviours among their children [39] compared to a control group receiving a community standard of care. Although there were no differences between the groups in terms of caregiver level of stress, FMF families reported that their family needs were more often met and caregiver satisfaction was significantly higher than in the control group.

\section{Parent-child interaction training}

Belcher et al. [40] studied the efficacy of the Home-U-Go Safely (HUGS) program for infants with intrauterine drug and alcohol exposure. A trained community health nurse conducted 16 motherinfant home visits ( $n=79$; from 1 week to 18 months of age) to monitor infant health and growth and provide information and support on parenting. Children in the HUGS intervention showed more improvements in internalizing and externalizing behaviours (based on maternal report) as compared to the control group, although at 3 years of age, there were no significant group differences in IQ.

The parent-child interaction training (PCIT) is a short-term empirically supported intervention program to enhance parent-child relationships, improve social skills, implement a positive discipline program, and reduce problematic child behaviours through coaching both parents and children [41]. The PCIT was examined among parent/child dyads with FASD ( $\mathrm{n}=58$, aged 3 to 7 years), over 14 weekly sessions, 90 minutes each. Both the PCIT group and control group (who only received psycho-educational parent coaching), reported reductions in child behavioural problems and parent satisfaction with the intervention, and the PCIT group showed a nonsignificant trend of more improvement compared with the control group [39]. This research suggests that both approaches have their merits and further inquiry is needed to determine a best practice in this regard.

\section{Youth outreach}

The Youth Outreach Program (YOP) is an intervention designed to support school success, community engagement, family relations, as well as reduced substance abuse, maladaptive behaviours, and criminal involvement among youth aged 13 to 18 years who have behaviours or characteristics consistent with FASD. Through formative evaluation and interviewing of 58 YOP stakeholders (youth, family members, program staff, and community partners), Hubberstey and colleagues [42] reported that the 3-year program led to positive outcomes in terms of safety, relationships, sexual health, substance abuse, school attendance, and awareness and access of community resources.

\section{Gaps}

Improving systems of support is clearly a critical part of intervention initiatives for behavioural needs identified in individuals with FASD. At this time, there is only very preliminary research 
focused on adolescent or adult supports, risk reduction in areas of delinquent behaviours or justice involvement, and direct intervention approaches geared towards increasing an individual's capacity for increased behavioural management or decision-making.

\section{Sensory-motor functioning}

\section{Needs}

Sensory-motor processing in FASD is another area of documented difficulty in FASD. Researchers have reported that deficits in sensory processing and sensory-motor functioning are related to adaptive behaviour and academic performance in home and school settings among children with FASD $[43,44]$. Impairments in sensory-motor integration and motor development have been noted in children and youth with FASD, with particularly pronounced difficulties in younger children [45].

\section{Sensory-motor interventions}

The empirical evidence for sensory-motor interventions in FASD is limited, and there are no studies to date that focus specifically on this area of need. Some preliminary work is being conducted to engage motor skills and build cognitive capacity among children aged 6 to 16 years with FASD through the use of video games [46], however no articles from these initiatives have yet been published. According to Jirikowic [47], interventions in this domain are typically embedded within larger interventions, making it difficult to identify outcomes specific to sensory-motor processing.

\section{Gaps}

Sensory-motor functioning appears to be one of the most neglected areas of treatment in FASD, with no intervention articles published in this area of need. Given that the sensory-motor processing abilities of children with FASD impact development, behaviour, and day-to-day functioning [47], it will be important for intervention researchers to focus on ways in which these skills can be strengthened in children and adults with FASD.

\section{Mental health}

\section{Needs}

Individuals with FASD have an increased susceptibility to mental health disorders, and this vulnerability persists across the lifespan $[6,13]$; over $90 \%$ of the FASD population is thought to experience comorbid mental health problems [48]. Most commonly, individuals with FASD experience depression and anxiety, attention-deficit hyperactivity disorder (ADHD), and conduct disorder (CD), but posttraumatic stress disorder, obsessive-compulsive disorder, oppositional defiance disorder, and bipolar disorder are also reported [48]. The nature of mental health issues in this population may change across the lifespan with alcohol and drug abuse being a serious concern for adults [48]. Compounding this risk is the fact that adults with FASD rarely access mental health services [49].

\section{Mental health interventions}

Despite the notable prevalence of co-morbid mental health problems among individuals with FASD, the published research on FASD-specific mental health interventions is limited, and what does exist is primarily medical in nature.

\section{Medical interventions}

All of the published research on medication for mental health symptoms in FASD has focused on ADHD. Snyder et al. [50] first examined the efficacy of stimulant medication on 11 children (aged 6 to 16 years) with FASD and co-morbid ADHD, and found that with stimulant medication, performance on an attention task did not significantly improve, but parent-ratings of hyperactivity did show improvements. Similarly, Oesterheld et al. [51] found that among four children with FASD and ADHD (aged 5 to 12 years), hyperactivity scores on a parental rating scale of attention improved with stimulant medication. Both of these studies with medication and FASD were limited by very small sample sizes that impacts power and generalizability, as well as insufficient information on dosing and medication compliance.

Doig et al. [52] examined the effects of medication on ADHD symptoms in a larger sample $(n=27)$ of children (aged 5 to 14 years) with FASD and found that children with FASD tended to showed more normalized scores on teacher-rated hyperactivity/impulsivity and opposition/defiance than inattention, indicating a different pattern of response than is reported for otherwise typically developing children.

\section{Gaps}

Considering the high prevalence of mental health problems among individuals with FASD, there is a surprising lack of research examining interventions to support mental well-being in this group. Specifically, researchers have focused solely on the use of stimulant medication to treat ADHD symptoms, neglecting other types of medication and other forms of mental illness such as anxiety or depression. Furthermore, there is a crucial gap in the literature for non-medical therapeutic interventions for individuals with FASD and comorbid mental health problems, and no published research to date on mental health supports for older adolescents or adults with FASD. Finally, considering that the mental health difficulties of individuals with FASD are thought to change across the lifespan, longitudinal research may shed light onto the developmental trajectories of this area of need.

\section{Adaptive functioning}

\section{Needs}

Many individuals with FASD experience significant difficulties with adaptive functioning including social-emotional functioning [53]. Children and adolescents with FASD have been shown to have immature psychosocial functioning [54], challenges with various social and interpersonal skills [55], and difficulties with communication, socialization, personal, and community skills [12]. These adaptive impairments in FASD persist and may even worsen with age, with adolescents and adults experiencing serious impairments in daily living skills, socialization, and communication, and problems with maladaptive behaviours [49].

\section{Adaptive interventions}

Although there is a consensus among researchers and clinicians that adaptive functioning is an area of significant impairment in FASD, efforts to address this need are few, and limited to basic safety and social interactions among children. 


\section{Social skills}

\section{Needs}

O'Connor et al. [56] studied the impact of a child friendship training (CFT) program on the social skills of 100 children with FASD (aged 6 to 12 years). Children in the CFT group demonstrated improvements in appropriate social behaviours and decreased problem behaviours (based on parental reports) compared to the control group, however, teachers did not make the same positive observations [56]. In a related study, Keil and colleagues [57] found that children receiving the CFT social skills intervention reported a reduction in their hostile attributions (although this was not reflected in reduced peer provocation), and reductions were maintained at 3-month follow-up.

\section{Medical interventions}

Frankel et al. [58] examined stimulant and neuroleptic medication use among 77 children (aged 5 to 11 years) with FASD participating in the CFT program. Children taking neuroleptic medication demonstrated greater improvement on parent- and teacher-ratings of social skills than those not taking neuroleptics. However, children taking stimulant medication did not show improvement (or showed worse outcomes) relative to those not taking stimulants.

\section{Safety}

Coles et al. [33] examined the efficacy of using a virtual reality computer game to teach safety skills to young children with FASD (aged 4 to 10 years) in either fire $(n=16)$ or street safety $(n=16)$ training. The authors found that both groups experienced knowledge gain in the content they were taught. Importantly, the majority of children (72\%) were able to generalize their learning to a more "real world" behavioural setting.

\section{Gaps}

Some promising gains have been made in developing interventions to improve social and safety skills among children with FASD, but again, this research is limited to children 12 years and younger and no studies involve longitudinal follow-up beyond 3 months. Given the significant adverse outcomes and functional difficulties commonly experienced by adolescents and adults with FASD [59], more research is needed to support daily living skill-building in these older individuals. Moreover, other adaptive abilities such as self-care, functional communication and literacy, money and household management, accessing community resources, and vocational skills are required for successful daily functioning but interventions to support these skills have not been examined in the FASD literature. Another significant gap is research into interventions designed to support transitional periods (e.g., from adolescence to adulthood), which may be a crucial window for intervention and improved by strong adaptive functioning [60].

\section{Caregiver considerations}

\section{Needs}

The impact of FASD extends beyond the affected individual and can put significant stress on the family, as well as strain on the relationships between caregivers and affected individuals [12]. Caregivers of those with FASD have reported concerns about self-care limitations and a lack of social and financial supports, a structured home setting, support from professionals, social involvement for both the child and their families, programs for managing difficult child behaviours, understanding of FASD, and inter-system collaboration [61,62]. Importantly, developmental outcomes for individuals with FASD depend on the quality of the caregiving environment as well as a functional and stable family unit [11]. Given the impact of FASD on the family and the vital role that family plays in achieving successful outcomes, researchers have asserted that it is crucial to provide support for caregivers. In particular, it is especially important to educate caregivers regarding the nature of FASD, support them in advocating for the person affected [14], and equip them with strategies to support positive outcomes [63].

\section{Caregiver-based interventions}

Olson et al. have hypothesized that fostering development of protective factors such as caregivers' level of optimism, caregiver efficacy, use of specialized caregiving practices, advocacy skills, and knowledge of FASD are important in providing appropriate support to a child with FASD. However, there are only a small number of evidence-based interventions for caregivers of individuals with FASD.

\section{Online education}

Kable et al. [63] evaluated the effectiveness of delivering educational materials to caregivers of children with FASD on-line, compared to community standard information packets and group workshops. Only the web-based and workshop groups made significant gains in knowledge about FASD and caregiver advocacy, while only the community and workshop groups led to positive behaviour gains.

\section{Mentoring supports}

Providing caregivers with mentoring supports is another strategy. Leenaars, Denys, Henneveld, and Rasmussen [64] examined the efficacy of Coaching Families [ $\mathrm{n}=186]$, a mentoring program for caregivers that provides support, education, advocacy, and referrals to families of children diagnosed with FASD. A significant decrease in reported family needs and caregiver stress, as well as an increase in goal-achievement was found from pre- to post-program. Caregivers also reported high overall satisfaction with the program.

Grant et al. [65] examined a mentoring program for women with FASD [ $n=19]$ to help connect them with community resources, obtain formal diagnoses of FASD, and assist with parenting. Grant et al. [65] found improved outcomes in the areas of substance use, contraceptive use, use of medical and mental health services, and improvements in maintaining stable housing arrangements. In a related study, Denys et al. [66] found that a similar mentoring program for parents with FASD $[n=24]$ was effective in helping clients reduce their needs and achieve their goals, particularly in the areas of social problems, experience of abuse, housing, transportation, self-care, and health.

\section{Gaps}

The existing literature on interventions for caregiver of individuals with FASD reveals perceived positive outcomes on the part of caregivers. However, research is lacking in terms of understanding the impact of such interventions on the relationship between the caregiver [or siblings] and the individual affected by FASD, as well as whether or how the effects of these interventions generalize to other systems such as the school setting or community services. Longitudinal research is needed to examine the long-term impact of caregiver interventions. As 
Page 6 of 9

well, because many adolescents and adults with FASD struggle with independent living [5], support for caregivers of older individuals with FASD is another important area to explore with future research.

\section{Discussion}

\section{Challenges}

Despite the well-documented needs and high costs of FASD, research into effective interventions continues to lag. Several calls for evaluation and inquiry into effective intervention have been made [67], which has catalyzed work in this area, but high costs and long timelines for intervention research slow the process of evidence accumulation. In a systematic review of interventions for children and youth affected by FASD, Premji and colleagues [10] identified problems with small sample sizes and weak research design. Nonetheless, access to interventions, particularly early in life, has been recommended as a protective factor for children with FASD [5], leading many professionals and service providers to establish programming for them. Unfortunately, beyond subjective assessment, formal evaluation of these initiatives is just beginning, and so information to guide further practice or program evolution is still in its infancy. Thus, both researchers and practitioners continue to call for further information to direct best practices. The present review has identified specific research gaps that exist in the literature, from which we can identify ways to move forward with our work.

\section{Specific intervention gaps}

\section{Interventions for adolescents and adults}

It is clear from this review that the majority of interventions for individuals with FASD target school-aged children. With the exception of research on caregivers with FASD, and a program evaluation for youth outreach, there are no published intervention studies focusing on adolescents or adults, despite the evidence that these are particularly challenging developmental stages. Unfortunately, the primary needs for individuals with FASD persist and may worsen as these individuals age. During adolescence, individuals with FASD often encounter extremely pronounced secondary disabilities including disrupted school experience, trouble with the law, victimization, and alcohol and drug abuse [5]. The gap in research on adolescent interventions is disconcerting given evidence to suggest that some of the cognitive impairments and decision making abilities among individuals with FASD may worsen with age and/or become exacerbated during adolescence [68,69], making this a particularly vulnerable time for individuals with FASD.

Adults with FASD continue to experience difficulties and require high levels of support and assistance. Streissguth et al. [5] found that among individuals with FASD [ $n=415] 83 \%$ of adults [21 years of age and older] were unable to live independently, citing issues such as poor planning, poor decision making, and an inability to follow through as key areas of concern. These challenges underscore the importance of appropriate and effective external supports and empirically-validated interventions. Of significant concern is the fact that once an affected individual passes the age of 18 , they are no longer eligible for pediatric services, and there are societal expectations that they function as adults and even live independently. With the developmental abilities of affected adults often lagging severely behind their chronological age, appropriate interventions and supports become all the more critical.

\section{Justice-based interventions}

One area of research that is growing rapidly is the association between FASD and justice-system involvement. Longitudinal studies suggest that $60 \%$ of youth and adults with FASD experience trouble with the law [6], and there is a remarkably high prevalence of FASD in both youth and adult correctional settings $[37,38]$. Individuals with FASD who enter the justice system are more likely than other offenders to have a high number of convictions [70], may become vulnerable targets for victimization, and are often unable to conform to the custodial environment [71]. It is recognized that special attention is required to address the unique and complex needs of justice-involved individuals with FASD. However, there is limited research on outcomes for these individuals [70] and currently no evidence-based interventions for this group.

\section{Emerging medical needs}

Another area for research growth is related to describing the physical needs present in FASD. In particular, nutritional deficits, sleep disorders, seizures, immune system vulnerabilities, elimination problems, and dental health concerns [72] are reported for this population, and identified as contributing to potential adverse outcomes. As such, future intervention work will ideally take a 'whole body' perspective, including brain and body function in considering impacts to daily functioning.

\section{Moving forward}

FASD is a complicated disorder that presents many unique challenges. To date, the most common approach to addressing these issues has been insular, deconstructive, and singular. However, Hyter and Way [73] suggested that singular disciplines cannot effectively or adequately address the complex issues and needs of individuals with $\mathrm{PAE}$, and what is needed are multitier interventions that consider more than the individual alone. The diverse needs and situations of individuals with FASD complicate the evaluation of interventions and outcomes, and raise the question of how to establish global outcomes that are meaningful across settings and individuals. For instance, Freisthler et al. [74] recommended that research efforts with individuals with disabilities include caregivers, families, and communities, as well as interactions between the individual and the environment in order to develop more holistic strategies that are effective in fostering positive outcomes in real world settings. It will also be important to learn from research and practice currently being implemented in other countries, cross-culturally, and across disciplines, to identify aspects of these practices that may apply or be adapted to the FASD population [73].

Other areas of research, such as literature related to childhood trauma as one example, may also provide valuable insight into how to respond to the needs of individuals with FASD. In addition to the neurocognitive effects of PAE, individuals with FASD may also experience high rates of living in an unstable home environment and exposure to abuse and trauma [5], which may place them in "double jeopardy" [11]. Putnam [75] suggested that the experience of childhood trauma affects core regulatory systems, which results in a compromised ability to modulate and process sensory stimuli and could render the child incapable of self-regulation, which is much like the behavioural phenotype as seen in FASD. Recognizing the impact of trauma, it follows that it is necessary to create an environment of psychological and physical safety, which provides a scaffold for skill building and affect management within the individual [76]. Shifting 
our understanding of behavior to the perspective that it is a manifestation of brain function that exists within an environmental context, introduces new ways of looking at intervention development and implementation from a unified perspective and can be applied to the FASD population. From this perspective, both the individual and the environment are malleable and may respond to interventions producing an overall gestalt effect of positive change.

\section{Conclusion}

Although 40 years of research has provided an increased understanding of the needs of individuals with FASD, efforts to respond to these identified needs have not yet received the same empirical inquiry. Fortunately, this appears to be changing, as research in the field of interventions is growing. O'Connor and Paley [77] recommended an exploration of how existing treatments may be adapted to suit the FASD population, ways to evaluate efficacy of multi-level approaches that attempt to respond to the broad range of issues present for those with FASD, and how to effectively translate research into practice.

There is general agreement that collaboration between disciplines will facilitate research that may provide insight into function across skill sets and settings. It is only through combined research efforts that the evidence supporting best practices will be established, and policies may be implemented to reflect best uses of available resources. As this progresses researchers can continue to examine outcomes in increasingly systematic ways, with increased use of meta-ethnographic and meta-analytic approaches. As the field of FASD continues to grow, so must the quality and quantity of research into intervention practices, not only so that practice can continue to evolve towards increasingly effective approaches, but also to provide the necessary evidence to support a system wide shift in the conceptualization and construction of interventions for the FASD population. This shift will be critical for meeting the complex and changing needs of these individuals and their families across the life span.

\section{Declaration of Interest}

The authors disclose no financial or personal relationships with other people or organizations that could inappropriately influence this work, and no affiliation with any organization with a financial interest, direct or indirect, in the subject matter or materials discussed in the manuscript.

\section{References}

1. Chudley AE, Conry J, Cook JL, Loock C, Rosales T, et al. (2005) Fetal alcohol spectrum disorder: Canadian guidelines for diagnosis. CMAJ 172: S1-1S21.

2. May PA, Gossage JP, Kalberg WO, Robinson LK, Buckley DG, et al. (2009) The prevalence and epidemiologic characteristics of FASD from various research methods with an emphasis on in-school studies. Dev Disabil Res Rev 15: 176-192.

3. Stade B, Ali A, Bennett D, Campbell D, Johnston M, et al. (2009) The burden of prenatal exposure to alcohol: revised measurement of cost. Can J Clin Pharmacol 16: e91-102.

4. Thanh NX, Jonsson E, Salmon A, Sebastianski M (2014) Incidence and prevalence of Fetal Alcohol Spectrum Disorder by sex and age group in Alberta, Canada. J Popul Ther Clin Pharmacol 2: e395-404.

5. Streissguth A, Barr H, Kogan J, Bookstein F (1996) Understanding the occurrence of secondary disabilities in clients with fetal alcohol syndrome
(FAS) and fetal alcohol effects (FAE). Seattle, WA: University of Washington.

6. Streissguth AP, Bookstein FL, Barr HM, Sampson PD, O'Malley K, et al. (2004) Risk factors for adverse life outcomes in fetal alcohol syndrome and fetal alcohol effects. J Dev Behav Pediatr 25: 228-238.

7. Olson H, Jirikowic T, Kartin D, Astley S (2007) Responding to the challenge of early intervention for fetal alcohol spectrum disorders. Infants and Young Children 20: 172-189.

8. Burd L (2006) Interventions in FASD: We must do better. Child Care Health and Development 33: 398-400.

9. Kodituwakku PW, Kodituwakku EL (2011) From research to practice: an integrative framework for the development of interventions for children with fetal alcohol spectrum disorders. Neuropsychol Rev 21: 204-223.

10. Premji S, Benzies K, Serrett K, Hayden KA (2007) Research-based interventions for children and youth with a Fetal Alcohol Spectrum Disorder: revealing the gap. Child Care Health Dev 33: 389-397.

11. Olson HC, Oti R, Gelo J, Beck S (2009) "Family matters:" fetal alcohol spectrum disorders and the family. Dev Disabil Res Rev 15: 235-249.

12. Paley B, O'Connor MJ (2011) Behavioral interventions for children and adolescents with fetal alcohol spectrum disorders. Alcohol Res Health 34: 64-75.

13. Burd L, Christensen T (2009) Treatment of fetal alcohol spectrum disorders: are we ready yet? J Clin Psychopharmacol 29: 1-4.

14. Paley B, O'Connor MJ (2009) Intervention for individuals with fetal alcohol spectrum disorders: treatment approaches and case management. Dev Disabil Res Rev 15: 258-267.

15. Jirikowic T (2003) Sensory processing and integration and children with alcohol related diagnosis: An explanatory analysis. Unpublished doctoral dissertation. University of Washington, Washington, DC.

16. Kodituwakku PW (2009) Neurocognitive profile in children with fetal alcohol spectrum disorders. Dev Disabil Res Rev 15: 218-224.

17. Mattson SN, Riley EP (1998) A review of the neurobehavioral deficits in children with fetal alcohol syndrome or prenatal exposure to alcohol. Alcohol Clin Exp Res 22: 279-294.

18. Santostefano S (1984) Cognitive control therapy with children: rationale and technique. Psychotherapy 21: 76-91.

19. Adnams CM, Sorour P, Kalberg WO, Kodituwakku P, Perold MD, et al. (2007) Language and literacy outcomes from a pilot intervention study for children with fetal alcohol spectrum disorders in South Africa. Alcohol 41: 403-414.

20. Riley EP, Mattson SN, Li TK, Jacobson SW, Coles CD, et al. (2003) Neurobehavioral consequences of prenatal alcohol exposure: An international perspective. Alcohol Clin Exp Res. 27: 362-373.

21. Williams MS, Shellenberger S (1996) “How does your engine run?" A leader's guide to the Alert Program for self- regulation. Albuquerque, NM: Therapy Works.

22. Wells AM, Chasnoff IJ, Schmidt CA, Telford E, Schwartz LD (2012) Neurocognitive habilitation therapy for children with fetal alcohol spectrum disorders: An adaptation of the Alert Program ${ }^{\circ}$. Am J Occup Ther 66: 24-34.

23. Nash K, Stevens S, Greenbaum R, Weiner J, Koren G, et al. (2015) Improving executive functioning in children with fetal alcohol spectrum disorders. Child Neuropsychol 21: 191-209.

24. Soh DW, Skocic J, Nash K, Stevens S, Turner, et al. (2015) Self-regulation therapy increases frontal gray matter in children with fetal alcohol spectrum disorder: evaluation by voxel-based morphometry. Front Hum Neurosci 9: 1-12.

25. Loomes C, Rasmussen C, Pei J, Manji S, Andrew G (2008) The effect of rehearsal training on working memory span of children with fetal alcohol spectrum disorder. Res Dev Disabil 29: 113-124.

26. Kerns K, MacSween J, Vander Wekken S, Gruppuso V (2010) Investigating the efficacy of an attention training programme in children with foetal spectrum disorder. Dev Neurorehabil 13: 413-422. 
27. Coles CD, Brown RT, Smith IE, Platzman KA, Erickson S, et al. (1991) Effects of prenatal alcohol exposure at school age. I. Physical and cognitive development. Neurotoxicol Teratol 13: 357-367.

28. Goldschmidt L, Richardson GA, Cornelius MD, Day NL (2004) Prenatal marijuana and alcohol exposure and academic achievement at age 10. Neurotoxicol Teratol 26: 521-532.

29. Rasmussen C, Bisanz J (2009a) Exploring mathematics difficulties in children with Fetal Alcohol Spectrum Disorders. Child Development Perspectives 3: 125-130.

30. Olson H, Burgess D, Streissguth A (1992) Fetal alcohol syndrome (FAS) and fetal alcohol effects (FAE): A life span view, with implications for early intervention. Zero-Three 13: 24-29.

31. Spohr HL, Willms J, Steinhausen HC (2007) Fetal alcohol spectrum disorders in young adulthood. J Pediatr 150: 175-179, 179.

32. Green JH (2007) Fetal Alcohol Spectrum Disorders: understanding the effects of prenatal alcohol exposure and supporting students. J Sch Health 77: 103-108.

33. Kable JA, Coles CD, Taddeo E (2007) Socio-cognitive habilitation using the math interactive learning experience program for alcohol-affected children. Alcohol Clin Exp Res 31: 1425-1434.

34. Coles CD, Kable JA, Taddeo E (2009) Math performance and behavior problems in children affected by prenatal alcohol exposure: intervention and follow-up. J Dev Behav Pediatr 30: 7-15.

35. Kable JA, Taddeo E, Strickland D, Coles CD (2015) Community translation of the Math Interactive Learning Experience Program for children with FASD. Res Dev Disabil 39: 1-11.

36. Mattson SN, Riley EP (2000) Parent ratings of behavior in children with heavy prenatal alcohol exposure and IQ-matched controls. Alcohol Clin Exp Res 24: 226-231.

37. MacPherson PH, Chudley AE, Grant BA (2011) Fetal Alcohol Spectrum Disorder (FASD) in a correctional population: Prevalence, screening and characteristics. Ottawa, ON: Correctional Service of Canada.

38. Popova S, Lange S, Bekmuradov D, Mihic A, Rehm J (2011) Fetal Alcohol Spectrum Disorder prevalence estimates in correctional systems: A systematic literature review. Can J Public Health 102: 336-340.

39. Bertrand J (2009) Interventions for children with fetal alcohol spectrum disorders (FASDs): Overview of findings for five innovative research projects. Res Dev Disabil 30: 986-1006.

40. Belcher H, Butz A, Wallace P, Hoon A, Reinhardt E, et al., (2005) Spectrum of early intervention services for children with intrauterine drug exposure. Infants and Young Children 18: 2-15.

41. Eyberg SM, Boggs SR (1998) Parent-child interaction therapy for oppositional preschoolers. In: Handbook of parent training: Parents as co-therapists for children's behavior problems. CE Shaefer and JM Briesmeister (Edtrs.) Wiley, New York.

42. Hubberstey C, Rutman D, Hume S (2014) Evaluation of a three-year Youth Outreach Program for Aboriginal youth with suspected Fetal Alcohol Spectrum Disorder. International Journal of Alcohol and Drug Research 3: 63-70.

43. Carr JL, Agnihotri S, Keightley M (2010) Sensory processing and adaptive behavior deficits of children across the Fetal Alcohol Spectrum Disorder continuum. Alcohol Clin Exp Res 34: 1022-1032.

44. Jirikowic T, Olson HC, Kartin D (2008) Sensory processing, school performance, and adaptive behavior among young school-aged children with FASD. Phys Occup Ther Pediatr 28: 117-136.

45. Williams L, Jackson CPT, Choe N, Pelland L, Scott SH, et al., (2014) Sensory-motor deficits in children with fetal alcohol spectrum disorder assessed using a robotic virtual reality platform. Alcohol Clin Exp Res 38: 116-125.

46. Bertram C, Mandryk R, Keiver K, Pritchard Orr A, Khaleel B, et al., (2012) Video games as therapy: The impact of a novel neurofeedback intervention for children with Fetal Alcohol Spectrum Disorders (FASD). Journal of Sport and Exercise Psychology 34 (Suppl.): S150-S194.

47. Jirikowic T (2013) Sensory processing and FASD. Presented at FASD: Best Practices in the Last Frontier. Anchorage, AK.
48. Pei J, Denys K, Hughes J, Rasmussen C (2011) Mental health issues in fetal alcohol spectrum disorder. J Ment Health 20: 438-448.

49. Streissguth AP, Aase JM, Clarren SK, Randels SP, Ladue RA, et al., (1991) Fetal Alcohol Syndrome in adolescents and adults. JAMA 265: 19611967.

50. Snyder J, Nanson J, Snyder R, Block G (1997) A study of stimulant medication in children with FAS. Seattle, WA: University of Washington Press.

51. Oesterheld J, Kofoed L, Tervo R, Fogas B, Wilson A, et al., (1998) Effectiveness of methylphenidate in Native American children with fetal alcohol syndrome and attention deficit/hyperactivity disorder: A controlled pilot study. J Child Adolesc Psychopharmacol 8: 39-48.

52. Doig J, McLennan JD, Gibbard WB (2008) Medication effects on symptoms of attention-deficit/hyperactivity disorder in children with fetal alcohol spectrum disorder. J Child Adolesc Psychopharmacol 18: 365-371.

53. Greenbaum RL, Stevens SA, Nash K, Koren G, Rovet J (2009) Social cognitive and emotion processing abilities of children with Fetal Alcohol Spectrum Disorders: A comparison with Attention Deficit Hyperactivity Disorder. Alcohol Clin Exp Res 33: 1656-1670.

54. Schonfeld AM, Paley B, Frankel F, O'Connor MJ (2006) Executive functioning predicts social skills following prenatal alcohol exposure. Child Neuropsychol 12: 439-452.

55. Kully-Martens K, Denys K, Treit S, Tamana S, Rasmussen C (2012) A review of social skills deficits in individuals with Fetal Alcohol Spectrum Disorders and prenatal alcohol exposure: Profiles, mechanisms, and interventions. Alcohol Clin Exp Res 36: 568-576.

56. O'Connor MJ, Frankel F, Paley B, Schonfeld AM, Carpenter E, et al. (2006) A controlled social skills training for children with fetal alcohol spectrum disorders. J Consult Clin Psychol 74: 639-648.

57. Keil V, Paley B, Frankel F, O'Connor MJ (2010) Impact of a social skills intervention on the hostile attributions of children with prenatal alcohol exposure. Alcohol Clin Exp Res 34: 231-241.

58. Frankel F, Paley B, Marquardt R, O'Connor M (2006) Stimulants, neuroleptics, and children's friendship training for children with fetal alcohol spectrum disorders. J Child Adolesc Psychopharmacol 16: 777-789.

59. Temple V, Shewfelt L, Tao L, Casati J, Klevnick L (2011) Comparing daily living skills in adults with Fetal Alcohol Spectrum Disorder (FASD) to an IQ-matched clinical sample. J Popul Ther Clin Pharmacol 18: e397-e402.

60. Lynch M, Kable J, Coles C (2015) Prenatal alcohol exposure, adaptive function, and entry into adult roles as a prospective study of young adults Neurotoxicol Teratol 5: 52-60.

61. Brown JD, Bednar LM (2004) Challenges of parenting children with fetal alcohol spectrum disorder: A concept map. Journal of Social Work 8: $1-18$.

62. Brown JD, Sigvaldason N, Bednar LM (2005) Foster parent perceptions of placement needs for children with a fetal alcohol spectrum disorder. Children and Youth Services Review 27: 309-327.

63. Kable J, Coles CD, Strickland D, Taddeo E (2012) Comparing the effectiveness of on-line versus in-person caregiver education and training for behavioral regulation in families of children with FASD. International Journal of Mental Health and Addiction 10: 791-803.

64. Leenaars L, Denys K, Henneveld D, Rasmussen C (2012) The impact of Fetal Alcohol Spectrum Disorders on families: Evaluation of a family intervention program. Community Ment Health J 48: 431-435.

65. Grant TM, Erns CC, Streissguth A, Stark K (2005) Preventing alcohol and drug exposed births in Washington State: Intervention findings from three parent-child assistance program sites. Am J Drug Alcohol Abuse 31: $471-490$.

66. Denys K, Rasmussen C, Henneveld D (2011) The effectiveness of a community-based intervention for parents with FASD. Community Ment Health J. 
Citation: Pei J, Flannigan K, Walls L, Rasmussen C (2016) Interventions for Fetal Alcohol Spectrum Disorder: Meeting Needs Across the Lifespan. Int J Neurorehabilitation 3: 192. doi:10.4172/2376-0281.1000192

Page 9 of 9

67. Stratton K, Howe C, Battaglia F (1996) Fetal alcohol syndrome: Diagnosis, epidemiology, prevention, and treatment. Washington, DC: National Academy Press.

68. Rasmussen C, Bisanz J (2009) Executive functioning in children with Fetal Alcohol Spectrum Disorders: profiles and age-related differences. Child Neuropsychol 15: 201-215.

69. Kully-Martens K, Treit S, Pei J, Rasmussen C (2013) Affective decisionmaking on the Iowa Gambling Task in children and adolescents with Fetal Alcohol Spectrum Disorders. J Int Neuropsychol Soc 19: 137-144.

70. Public Health Agency of Canada (2007) Report: National roundtable on the development of a Canadian model for calculating the economic impact of FASD.

71. Conry J, Fast DK (2000) Fetal Alcohol Spectrum Disorder and the criminal justice system. Maple Ridge, BC: British Columbia Fetal Alcohol Syndrome Resource Society Vancouver and Law Foundation of British Columbia.

72. Hanlon-Dearman A, Green CR, Andrew G, LeBlanc N, Cook J (2015) Anticipatory guidance for children and adolescents with Fetal Alcohol
Spectrum Disorder (FASD): Practice points for primary health care providers. J Popul Ther Clin Pharmacol 22: e27-e56.

73. Hyter Y, Way I (2007) Understanding children who have been affected by maltreatment and prenatal alcohol exposure: Future directions. Lang Speech Hear Serv Sch 38: 157-159.

74. Freisthler B, Merritt DH, LaScala EA (2006) Understanding the ecology of child maltreatment: a review of the literature and directions for future research. Child Maltreat 11: 263-280.

75. Putnam FW (2003) Ten-year research update review: child sexual abuse. J Am Acad Child Adolesc Psychiatry 42: 269-278.

76. Henry J, Sloane M, Black-Pond C (2007) Neurobiology and neurodevelopmental impact of childhood traumatic stress and prenatal alcohol exposure. Lang Speech Hear Serv Sch 38: 99-108.

77. O'Connor M, Paley B (2009) Psychiatric conditions associated with prenatal alcohol exposure. Dev Disabil Res Rev 15: 225-234. 